POS PROCEEDINGS

\title{
Level 1 core software testing for Planck/LFI
}

\author{
Marco Frailis*, Michele Maris, Michele Guerrini, Andrea Zacchei, Fabio Pasian \\ Astronomical Observatory of Trieste \\ E-mail: frailiseoats.inaf.it
}

Reiner Rohlfs, Nicolas Morisset, Marc Türler

Geneva Astronomical Data Centre

Maurizio Miccolis, Paolo Leutenegger, Paola Battaglia, Cristian Franceschet

Alcatel Alenia Space Italia S.p.A. (Milano)

\begin{abstract}
The Level 1 of the PLANCK Low Frequency Instrument (PLANCK/LFI) Data Processing Centre (DPC) is devoted to the handling of scientific and housekeeping telemetry. It receives raw telemetry packets from the LFI through a tailored version of the ESA telemetry handling software SCOS 2000, and generates time ordered information of scientific and housekeeping data to be processed by the subsequent DPC levels. In addition, the Level 1 performs live diagnostics on the LFI telemetry. An end-to-end test has been performed recently at the LFI integration site (Alcatel Alenia Space, Milan) using the qualification model equipment of the on-board Data Acquisition Electronics and Data Processing unit, and its core software. For scientific data, the test is based on the injection of deterministic signals with known properties in the acquisition electronics and their comparison with the signal reconstructed from the telemetry data. A regression test has been performed on the housekeeping data by inserting known parameters values into a set of real housekeeping packets.
\end{abstract}

$C M B$ and Physics of the Early Universe

20-22 April 2006

Ischia, Italy

\footnotetext{
* Speaker.
} 


\section{The LFI Level 1 Software}

The ESA PLANCK satellite ${ }^{1}$ [1], scheduled for launch in 2008, is a full-sky surveyor dedicated to CMB and (sub)mm astronomy and represents a third generation mission after COBE and WMAP. PLANCK is equipped with a $1.5 \mathrm{~m}$ Gregorian aplanatic telescope, carrying in the focal surface two instruments covering the frequency bands 30, 44, and $70 \mathrm{GHz}$ (Low Frequency Instrument, LFI [沟) and 100, 143, 217, 353, 545, and $857 \mathrm{GHz}$ (High Frequency Instrument, HFI [3]). Each instrument will have its own dedicated Data Processing Centre (DPC) [ 4 ] in charge of retrieving the raw telemetry data, assessing their overall quality and the instrument health, performing data reduction and scientific analysis and releasing the final products to ESA. Each DPC breaks down the entire data processing into subsequent levels. The Level 1 (L1) of the LFI processing is formed by three subsystems: a tailored version of SCOS 2000, monitoring the housekeeping (HK) telemetry and sending the raw telemetry (TM) packets to the following subsystems; the Telemetry Handler System (TMH), which processes both scientific (SCI) and HK packets generating Time Ordered Information (TOI) for the subsequent DPC levels; the Telemetry Quick-Look System (TQL), displaying SCI and HK raw data and performing quick look analysis of SCI data. In particular, the TMH performs the acquisition and storage of the TM packets from SCOS 2000, time unscrambling operations, the decoding of TM packets, the conversion of the TM packets content into physical units and the creation of TOIs of SCI and HK data. The TQL is a "live" display of SCI and HK data as a function of time. For every selected detector, it shows different views of data as they are received. Among the possible views are strip charts, histograms, correlation plots, Fourier transforms, several statistical estimators.

\section{Tests objectives and hardware setup}

The focus of the end-to-end tests is the Qualification Model (QM) of the DPC L1 software as part of the testing campaign performed on the QM of the LFI. They have to assess the proper coverage of the requirements by the TMH/TQL and the validation of the TMH/TQL operations. The main testing scheme used is a forward comparison between the input data provided by the hardware and the output provided by the Level 1 software. The tests have been ordered according to a functional classification of the requirements, consisting of handling raw TM packets and time information, decompression, decoding and reconstruction of SCI and HK packet contents, TOIs generation and graphical display [5]. Input signals of known properties have been injected into the acquisition chain in order to test the behavior of the software system. Metrics have been applied to the output of TMH/TQL to assess the proper representation of the input. The complete acquisition chain of LFI (see figure 1) is implemented through a Digital Acquisition Electronic unit (DAE) and the Radiometer Electronic Box Assembly (REBA, delivered by the team of the Instituto de Astrofísica de Canarias). The first biases the radiometers (voltage drivers with relevant HK information) and digitises the radiometric output signal (PGA, Offset removal and A/D conversion). The digitised data are processed by the REBA in a scientific pipeline in order to reach the required compression efficiency. The pipeline downsamples the data, and performs statistical equalization, redigitisation, lossless compression, and generates the packets. Each step

\footnotetext{
${ }^{1}$ http://www.rssd.esa.int/planck
} 


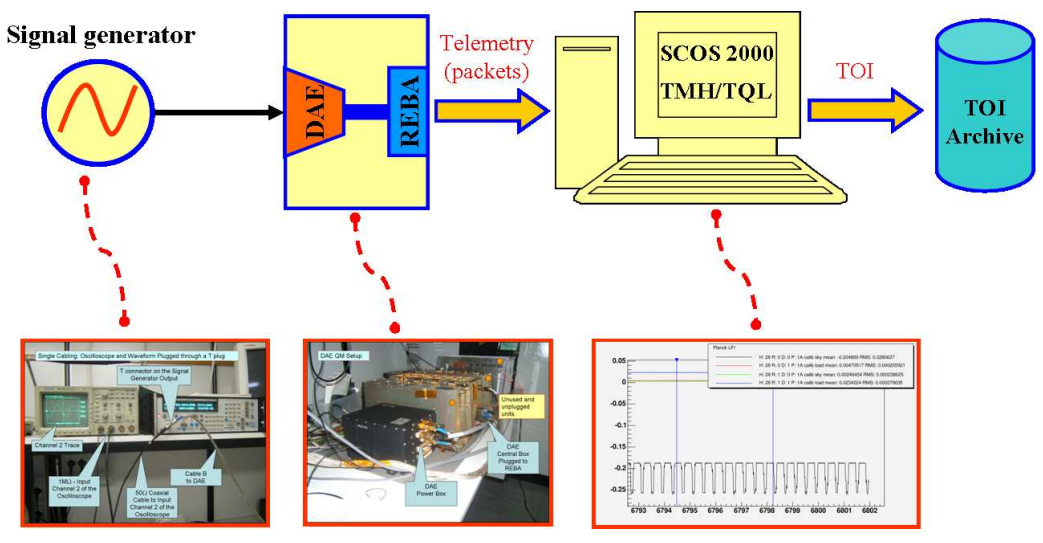

Figure 1: Hardware setup for the end-to-end tests

of this pipeline defines a particular diagnostic acquisition mode, which shall be properly handled by the L1 software. A calibrated waveform synthesizer has been used to generate signals of known shape and electrical characteristics which have been injected in selected channels of the acquisition chain.

\section{Scientific and housekeeping processing validation}

The proper processing of scientific data is assessed by quantitative comparison between the parameters of the input signal monitored by an oscilloscope with the corresponding data from the acquisition chain and the L1 software. Period, duty-cycle, lower and upper voltages, noise RMS, and shape of the signal have been measured on the data streams produced by each test. The ability of the REBA to output two different intermediate steps of its pipeline has been exploited during the tests. Data have been acquired changing alternatively signal properties or the setup of the on-board processing. We have checked for proper registration of input signals to the corresponding TOIs, proper shape reconstruction, time reconstruction by phase analysis and verifying the periods of the recorded signals using Lomb-Scargle periodogram, voltage reconstruction comparing high and low states in square waves, digitisation noise comparing input and output of the on-board redigitisation, and compression and decompression comparing the input and output of the compression/decompression stage. Among the obtained results, the tests have detected a problem in the TMH handling of some types of diagnostic data. They have assessed the RMS in period reconstruction to be $\approx 1 \mathrm{msec}$ (to be compared to the $\approx 7 \mathrm{msec}$ sampling time), measuring the ability of the full acquisition chain to properly estimate time intervals. Actions have been issued to solve the detected problems before the start of the Flight Model tests [6].

Differently from the scientific telemetry, it wasn't possible to inject known signals into the HK packets directly through the DAE/REBA chain. An Housekeeping Validation System (HVS) has been developed to manipulate the binary representation of an HK packet with the goal of generating packets with known parameter values starting from a set of real HK packets. For each HK packet type, the HVS system iterates over all the samples, setting to 1 one bit a time. Hence, for each sample, only a single bit of a single parameter is changed. This implies that for a given HK packet, 
REBA HK, TM(3,25), SID=202

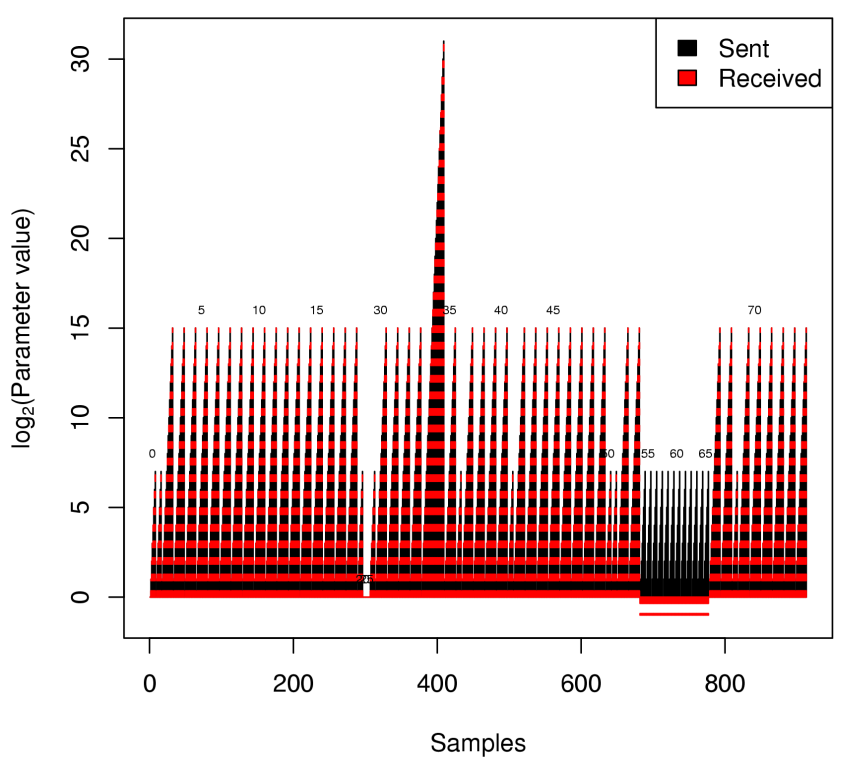

Figure 2: Comparison of testing values for REBA HK

each parameter in turn takes a value which increases by power of 2 . One of the purposes of this pattern is to verify that in the TMH system the offset and the length of each parameter has been correctly defined. The results of the HK tests have shown that the TMH properly handles the HK telemetry with the exception of one bug in the registration of HK spare parameters used in the REBA HK and REBA DIAGNOSTIC packets. The problem is shown in figure 2 , representing a comparison of testing values in HK parameters (black) with values in the corresponding TOIs (red). Parameters are ordered according to their position in the packet (denoted by the number on the top of each peak). The "sample" axis is the location in the test sequence where that sample is tested with the given value. The problem with the registration of HK parameters is evidenced by the anomalous distribution of red points around the sample 700. This test has proven that there is a discordance between the document describing the packets structure for LFI and their mapping in the SCOS 2000 mission information base [7].

\section{Conclusions}

The testing scheme described in this work, based on the use of a calibrated signal generator to feed the acquisition chain and the whole data reduction pipeline, turned out to be a valuable procedure to integrate the results obtained on simulated data. The LFI L1 software has been systematically validated and the problems detected by the end-to-end tests have already been solved. 


\section{References}

[1] J.A. Tauber, The Planck Mission, Advances in Space Research, Vol. 34, issue 3, pp. 491-496, 2004

[2] N. Mandolesi et al., Planck LFI, A Proposal Submitted to the ESA, 1998

[3] J.L. Puget et al., Hight Frequency Instrument for the Planck mission, A Proposal Submitted to the ESA, 1998

[4] F. Pasian, J.F. Sygnet, Planck data processing centres, Data Analysis II, SPIE proceedings, 4847, pp. 25-34, 2002

[5] M. Maris, M. Frailis, X. Dupac, Planck LFI Test Plan for the TMH software, Planck. Int. Rep. (PL-LFI-OAT-PL-09), 2005

[6] M. Frailis, M. Maris, M. Guerrini et al., LFI Level 1 software end-to-end tests started for the FM, The Planck Newsletter, issue 8, 2006

[7] M. Maris, M. Frailis, M. Guerrini, Planck LFI - Test Report on the TMH/QM by Using A Known Signal Tests Data, Planck. Int. Rep. (PL-LFI-OAT-RP-17), 2006 